\begin{abstract}
"Mircea cel Batran" Naval Academy Scientific Bulletin, Volume XIX - 2016 - Issue 1
Published by "Mircea cel Batran" Naval Academy Press, Constanta, Romania /I The journal is indexed in:

PROQUEST / DOAJ / DRJI / JOURNAL INDEX / I2OR / SCIENCE LIBRARY INDEX / Google Scholar / Crossref /

Academic Keys / ROAD Open Access / OAJI / Academic Resources / Scientific Indexing Services / SCIPIO
\end{abstract}

\title{
LE CINEMA HITCHCOCKIEN - APPROCHE GÉNÉRALE
}

\author{
Corina SANDIUC ${ }^{1}$ \\ ${ }^{1}$ Assistant Lecturer "Mircea cel Batran" Naval Academy
}

\begin{abstract}
II est impossible de surestimer l'influence d'Alfred Hitchcock sur l'histoire du cinéma. Très populaire à la fois pour le public et parmi les critiques, sa carrière prolifique s'est étendue sur cinq décennies et a produit des dizaines de films, dont de nombreux qui sont considérés comme des classiques inestimables. Sans doute un des plus habiles réalisateurs du XXème siècle, Hitchcock a été reconnu comme le maître du suspense cinématographique, un créateur qui a exercé un «contrôle presque pavlovien» sur les émotions et les réactions de son public. Ces émotions et le plasir qui en découle sont acquis par le biais des techniques cinématographiques et des procédures subliminales qu' Hitchcock contrôlait si bien. II pourrait vraiement captiver le public; c'est ainsi que sa capacité de manipuler les émotions des spectateurs est devenue légendaire.

Dans cet article on va aborder la conception artistique d'Hitchcock vis-à-vis l'art cinématographique comme véhicule du plaisir resultant de l'émotion, du suspense, et, de manière explicite, de la structure du montage, en s'appuyant, tout d'abord, sur ses interviews et ses déclarations et secondairement sur la critique qui lui a été dédiée.
\end{abstract}

Mots-clé : Alfred Hitchcock, cinéma, suspense, technique cinématographique;

Alfred Hitchcock a généralement donné des explications de son approche de la cinématographie. Dans beaucoup de ses déclarations, il parle de ses procédés méthodologiques tout en précisant la nature de ce qu'il réalise dans ses films. En ce sens, dans son long entretien avec François Truffaut il décrit sa mission en tant qu'artiste comme suit: «Le premier travail c'est de créer l'émotion et le deuxième travail c'est de la préserver. ${ }^{1}$ Cette émotion et le plasir qui en découle sont acquis par le biais des techniques cinématographiques et des procédures subliminales qu'Hitchcock contrôlait si bien. II pourrait vraiement captiver le public; c'est ainsi que sa capacité de manipuler les émotions des spectateurs est devenue légendaire. Son succès inégalé, est mesuré par le fait que ses films continuent à fasciner des millions de personnes dans le monde entier. Compte tenu de cette intention de manipulation, le travail d'Hitchcock a été considéré comme «le produit d'un Frankenstein ou d'un proto-fasciste qui est extrêmement talentueux à susciter des réactions affectives par le biais de la technologie cinématographique.» ${ }^{2}$

Un des théoriciens qui ont largement influencé Hitchcock a été Sergei Eisenstein. De son approche formaliste, Hitchcock a atteint une prise de conscience raffinée de la façon dont les effets cinématographiques influencent les réactions et les émotions du public. Eisenstein a cherché à utiliser les dispositifs artificiels du film comme un moyen de diffuser la perspective idéologique du

\footnotetext{
${ }^{1}$ François Truffaut, 1983, p. 92.

${ }^{2}$ Irving Singer, 2004, p.25.
}

directeur grâce à des techniques qui manipulent les sentiments du public. Comme d'autres formalistes de son époque, il a vanté la capacité des films de toucher l'esprit et la réactivité de leurs spectateurs. Eisenstein entend par cela formuler un programme politique par le biais de la technologie hypnotique du film, qui a comme but d'amener les gens à s'engager dans l'action qui aura une importance pour eux comme des êtres sociaux et moraux. ${ }^{3}$ Hitchcock ne pense pas à son public de cette façon. II le traite comme des simples individus qui peuvent être amenés à expérimenter des émotions fortes, mais il s'agit ici des émotions qui ont peu de pertinence pour les convictions politiques ou la participation communautaire. Hitchcock ne tente pas de donner ouvertement des idées de propagande, ce qu'il emprunte plus particulièrement d'Eisenstein c'est la conviction que l'utilisation subtile de la caméra par le cinéaste peut influencer les impulsions, et même les attitudes de n'importe qui observe le produit fini.

Dans un documentaire sur Hitchcock, l'un de ses associés, Ernest Lehman, celui qui a écrit le scénario de North by Northwest, raconte une conversation dans laquelle le directeur lui dit: «Ce que nous faisons, c'est que nous jouons, nous sommes assis à un organe et nous appuyons sur cet accord qui fait le public dire 'oooh », et ensuite nous appuyons sur cette corde qui les fait rire. Et nous jouons tous simplement avec eux.» ${ }^{4}$ Mais pour pour la faire vibrer, on doit tout d'abord trouver la corde sensible du spectateur et il

\footnotetext{
${ }^{3}$ Sergei Eisenstein, pp. 273-4.

${ }^{4}$ Ernest Lehman, p. 27. 


\begin{abstract}
"Mircea cel Batran" Naval Academy Scientific Bulletin, Volume XIX - 2016 - Issue 1
Published by "Mircea cel Batran" Naval Academy Press, Constanta, Romania /I The journal is indexed in: PROQUEST / DOAJ / DRJI / JOURNAL INDEX / I2OR / SCIENCE LIBRARY INDEX / Google Scholar / Crossref /

Academic Keys / ROAD Open Access / OAJI / Academic Resources / Scientific Indexing Services / SCIPIO
\end{abstract}

semble que cette corde sensible est «l'imaginaire qui se nourrit de désirs et de craintes. Parce qu'Hitchcock prend pour unique sujet et pour unique objet de son oeuvre l'angoisse qui fertilise l'imagination et l'imagination qui alimente l'angoisse, la menace qui plane en permanence sur ses personnages renvoie à celle éprouvée dans les affres de la création. ${ }^{5}$

La peur et les états psychiques conjoints: la menace et l'angoisse, font partie du monde complexe et trouble des sensations de l'individu, renvoyant à une époque quand on en dépendait pour nous assurer la survivance. Ils sont des sens qui, même si parfois assoupi, existent en nous et demandent l'expression de temps en temps. Non seulement ça, mais si l'existence d'aujourd'hui n'est plus tellement menacée par la menace basique, on se sait quand même menacé par une multitude de facteurs. L'angoisse fait partie de nos vies, chacun la connaît, c'est pourquoi y trouver la solution par l'intermède d'une fiction peut offrir le défoulement dont on a besoin. C'est tout comme si on vivait dans le monde subjectif de notre imagination ce qu'on ne peut pas vivre dans le monde objectif de la réalité.

Le monde subjectif et celui objectif lient des rapports complexes et multiples qui ont en commun l'existence elle-même. Chacun d'entre nous vit à un niveau subjectif aussi qu'à un niveau objectif. Un sans l'autre, c'est la division de l'être elle-même. La contradiction et l'opposition entre les deux restent à la base de l'oeuvre hitchcockien. L'existence de ces deux mondes justifie «les passages tantôt insensibles, tantôt brutaux, du monde objectif au subjectif, qui forcent le spectateur à s'embarquer dans l'univers irrationnel de la pure sensation ${ }^{6}$ Tout dans un film n'existe que sous l'angle de notre subjectivité et de l'émotion qu'il suscite en nous. C'est ainsi qu'Hitchcock nous introduit dans son univers pour en prouver l'existence. Sa seule préoccupation semble être nous couper du réel et nous faire pénétrer dans le suspense, car nous ressentons sa fantasme comme une réalité, qui peut être plaisante ou déplaisante.

Le public tient le rôle principal dans l'œuvre filmique, il représente «le monde objectif face au film, projection des fantasmes du cinéaste $\aleph^{7}$ Pendant la projection, le film semble le seul univers possible vrai. En se transportant dans le film, le spectateur renonce graduellement à l'état passif pour s'assumer celui d'acteur actif. Pour arriver à cela et ainsi obtenir la preuve de la meilleure participation du public, «il faut que

\footnotetext{
5 Jean Douchet, 1999, p. 52.

${ }^{6}$ Ibidem.

${ }^{7}$ Idem, p. 53.
}

l'œuvre, projection de l'univers mental du cinéaste coïncide exactement avec la projection mentale du public ; que les forces animées qui se meuvent sur l'écran expriment à la perfection de ses désirs et de ses craintes; qu'à la limite, le film qui se déroule sous ses yeux soit son film. ${ }^{8}$ On peut voir comment le spectateur est important dans la création d'un film, partie essentielle de celle-ci car l'imagination dont Douchet parle appartient non seulement à l'auteur, mais au récipient cible du film, c'est-à-dire au spectateur. C'est lui qui charge de significations les éléments du film qu'il filtre par son imagination, son intelligence, son esprit.

On aime ou on n'aime pas un film; deux personnes proches l'une de l'autre peuvent avoir des réactions différentes. Selon Christian Metz, pour aimer un film il faut: " assouvir les pulsions du sujet en flattant ses fantasmes » et « limiter l'assouvissement en-deçà du point d'angoisse et de rejet. ${ }^{9}$ Un détail suffit pour produire le déplaisir ou le plaisir insuffisant (la frustration), mais si le film a plu, il doit beaucoup aux fantasmes de son spectateur qui jouit d'une relation duelle avec lui.

Néanmoins, pour arriver à une telle immersion du public dans la subjectivité, dans la terreur de ses illusions, Hitchcock s'appuie sur des éléments rationnels: le montage et la logique de l'intrigue. Car on ne peut pas duper le public si facilement, on doit le séduire graduellement à ce qu'il croie à la réalité du monde cinématographique et ainsi « se laisser happer par la fiction », s'est à dire s'identifier aux personnages sur l'écran ou à la caméra dans ses mouvements complexes. ${ }^{10}$

Pour mieux comprendre comment Hitchcock a reussi à manipuler les émotions de ses spectateurs de telle manière que le film leur produise le plus grand plaisir, il suffit de se concentrer sur les remarques faites par lui à propos du montage dans plusieurs contextes. Tout comme Eisenstein il utilise le terme montage pour désigner sa technique de modifier la réponse du public par le biais de la communication cinématographique. II parle du montage comme de la base de ce qu'il appelle le cinéma pur, c'esta-dire un cinema qui créerait un monde clos independant des répères du realisme. Le cinéma pur est un concepte qui remonte aux débats théoriques et esthétiques de la critique française des années 1920, dont certains considéraient que le cinéma était une forme d'art, car il englobe tous les autres arts: la littérature, la musique, la danse, le théâtre, la peinture, la poésie et la

\footnotetext{
${ }^{8}$ Ibidem.

${ }^{9}$ Christian Metz, p. 136.

${ }^{10}$ Idem, p. 85.
} 


\begin{abstract}
"Mircea cel Batran" Naval Academy Scientific Bulletin, Volume XIX - 2016 - Issue 1
Published by "Mircea cel Batran" Naval Academy Press, Constanta, Romania /I The journal is indexed in: PROQUEST / DOAJ / DRJI / JOURNAL INDEX / I2OR / SCIENCE LIBRARY INDEX / Google Scholar / Crossref /

Academic Keys / ROAD Open Access / OAJI / Academic Resources / Scientific Indexing Services / SCIPIO
\end{abstract}

photographie, tandis que d'autres, les défenseurs du cinéma pur, insistaient que les films ont des caractéristiques uniques et spécifiques. Hitchcock a utilisé le terme d'une façon moins extrême, en faisant référence à la façon dont un directeur peut exprimer des pensées ou créer une situation dramatique, sans avoir besoin de paroles, simplement par le choix et l'arrangement des images. ${ }^{11}$

L'adhésion d'Hitchcock à la notion du cinéma pur se montre dans la délectation avec laquelle il décrit son habileté en tant que technicien. II est toujours préoccupé par les questions de la façon d'utiliser ses outils et la technologie disponible pour la réalisation de certains effets. La remarque qu'il cite lui-même comme ayant servi à aider les différents acteurs qui ont eu des problèmes dans leur exécution: «ll n'est qu'un film!» en dit long sur l'approche de son art. La réalité humaine qui entre dans la créativité du film à différents niveaux est, pour lui, subordonnée aux solutions techniques, qu'il a réussi à manoeuvrer avec adresse. Il ne voit pas le réel comme le but ultime que l'art doit chercher à pénétrer, à divulguer, ou encore à expliciter. Le monde dépeint par Hitchcock est ce que nous le rencontrons tous les jours, et pourtant, pour lui, il existe surtout comme quelque chose de notre expérience qui nous attire dans l'artificialité de la construction de son esthétique. Son art réside dans l'évocation des émotions qui sont habituellement silencieuses ou immergées dans la vie. ${ }^{12}$

Quand Hitchcock parle du but artistique de ses films, il dit: «Tourner des films pour moi veut dire d'abord, et avant tout, raconter une histoire. Cette histoire peut être invraisemblable mais elle ne doit jamais être banale. II est préférable qu'elle soit dramatique et humaine. Le drame c'est une vie dont on a éliminé les moments ennuyeux. Ensuite, la technique entre en jeu et, là, je suis ennemi de la virtuosité. II faut ajouter la technique à l'action. II ne s'agit pas de placer la caméra dans un angle qui provoquera l'enthousiasme du chef opérateur. La seule question que je me pose est de savoir si l'installation de la caméra à tel ou tel endroit donnera à la scène sa force maxima. La beauté des images, la beauté des mouvements, le rythme, les effets, tout endroit être soumis et sacrifié à l'action.» ${ }^{13}$

Ces principes sont très bien illustrés dans son explication au sujet de la scène de Sabotage dans laquelle Sylvia Sidney tue son mari:

«Lorsque Sylvia Sidney apporte le plat de légumes sur la table, elle est réellement hantée

\footnotetext{
${ }^{11}$ Marilyn Fabe, 2004, p. 140.

${ }^{12}$ Irving Singer, op. cit., p. 22.

${ }^{13}$ François Truffaut, op. cit., p. 83.
}

par le couteau, comme si sa main allait le saisir indépendamment de sa volonté. La caméra cadre sa main puis ses yeux jusqu'au moment ou son regard devient brusquement conscient de ce que le couteau signifie [...] Maintenant la caméra est sur Verloc, ensuite elle va vers le couteau et encore sur Verloc, vers son visage. Tout à coup, on comprend qu'il aperçoit le couteau et qu'il comprend ce que ce couteau veut dire pour lui. Le supense entre les deux personnages est créé, et, entre eux se trouve le couteau. À présent, grace à la caméra, le public fait partie de la scène et il ne faut surtout pas que la caméra devienne brusquement distante et objective, sous peine de détruire l'émotion qui a été créée. Verloc se lève et contourne la table mais, en le faisant, il marche directement vers la caméra, de façon que s'établisse dans la salle le sentiment qu'il faut reculer pour lui faire de la place; si cela est reussi, instinctivement, le spectateur doit reculer légerement dans son fauteuil pour laisser passer Verloc devant lui; lorsque Verloc est passé devant nous, la caméra glisse à nouveau vers Sylvia Sidney et elle retourne à l'objet principal, le couteau. Et la scène continue, jusqu'au meurtre.» ${ }^{14}$

Hitchcock explique sa dépendance à l'égard du montage dans ces scènes en parlant de la façon dont il peut faire le meurtre paraître inévitable, sans aucune faute attachée à la femme: «Tout le problème était là: il fallait que la sympathie du public reste acquise à Sylvia Sidney, il fallait que la mort de Verloc ne soit qu'un accident et pour cela, il était absolument nécessaire que le public s'identifie à Sylvia Sidney. Dans ce cas précis, on ne demande pas au public d'avoir peur, mais carrément d'avoir envie de tuer» ${ }^{15}$ Hitchcock affirme que cette révélation de la nature des sentiments requiert une approche de la part du réalisateur qui est radicalement différente de la procédure habituelle. Au lieu d'enregistrer une succession d'événements qui allaient être réduits et réunis dans la salle de coupe, le directeur doit avoir une conception préalable de la réponse qu'il souhaite atteindre et de la manière dont elle peut être évoquée. Plusieurs choses sont implicitement affirmées par cette méthode. Elle met sur le directeur une grande responsabilité car «le metteur en scène [...] est un dieu, il doit créer la vie.»16 || contrôle non seulement la forme générale du film qu'il fait mais tous les détails de son contenu et de sa construction aussi. Au lieu de reproduire le monde tel qu'il est ou comme une toile de fond pour raconter une histoire ou de

\footnotetext{
${ }^{14}$ François Truffaut, op. cit., p. 92.

15 Ibidem.

${ }^{16}$ Idem, p. 82.
} 


\begin{abstract}
"Mircea cel Batran" Naval Academy Scientific Bulletin, Volume XIX - 2016 - Issue 1
Published by "Mircea cel Batran" Naval Academy Press, Constanta, Romania /I The journal is indexed in: PROQUEST / DOAJ / DRJI / JOURNAL INDEX / I2OR / SCIENCE LIBRARY INDEX / Google Scholar / Crossref /

Academic Keys / ROAD Open Access / OAJI / Academic Resources / Scientific Indexing Services / SCIPIO
\end{abstract}

fournir un message, le directeur présente un événement dans la narration par l'intermédiaire d'un processus que seul le cinéma peut concevoir.

Raymond Bellour souligne aussi que les films d'Hitchcock reposent sur des scénarios d'une grande rigueur et qu'ils sont admirablement interprétés, mais que les intrigues ainsi que les comédiens sont soumis entièrement à la volonté du metteur en scène afin de créer l'impression que «que c'est par l'obsession de son auteur et son acharnement à regarder le monde que l'œuvre vit vraiment, on la doit à la caméra, à elle seule qui guette et sans cesse poursuit les mouvements des êtres et la forme des choses. ${ }^{17}$ Plus importante encore c'est l'affirmation d'Hitchcock que l'idéal analogique du film est le court récit. Dans une histoire courte la longueur empêche le spectateur de plonger trop profondément dans la réalité d'une situation. Sans être artistiquement superficiel, le court récit survole une surface assez longtemps pour qu'il piège et retienne l'intérêt du lecteur jusqu'à un point culminant évident a été atteint. Hitchcock a essayé d'éviter les adaptations des romans, car il pensait qu'ils ne peuvent pas être réduites à la plus petite échelle du film, et donc ne se prêtent pas à la transformation nécessaire du littéraire en visuel. Néanmoins, il a fait des adaptations de romans, et même de pièces de théâtre entières, par exemple, Corde et Le crime était presque parfait, mais il les a filmées comme des répliques de la mise-en-scène des spectacles, sans beaucoup de montage.

Le réalisateur choisit et ordonne les «petits morceaux» de matériel cinématographique en fonction de sa décision antérieure sur les motivations de certains personnages; il a imaginé l'incontrôlable sentiment de fatalité vécue par la femme en ce qui concerne le couteau qui va alors tuer son mari. Enfin, le directeur impose une interprétation, dans ce cas un jugement moral sur la culpabilité ou l'innocence de la femme. Elle doit être considérée comme luttant irréprochablement contre quelque chose de plus fort qu'ellemême. Tout ceci est organisé pour stimuler des sentiments particuliers dans le public. Les mouvements de la caméra nous poussent «à nous identifier à des personnages ou à des héros à la liberté factice.» ${ }^{18}$ Notre sympathie pour la meurtrière doit être préservée alors que nous restons plongés dans l'observation d'un événement brutal qui a sur nous un effet de délivrance cathartique qui s'ensuit de la situation particulière et de sa place dans la narration.

\footnotetext{
${ }^{17}$ Raymond Bellour, 1979, p. 47.

${ }^{18}$ Christian Metz, op. cit., p. 85.
}

Comme une nouvelle illustration des idées d'Hitchcock sur le montage, considérons l'épreuve de Tippi Hedren dans le grenier de la maison assiégée dans Les oiseaux. Après avoir lentement monté les marches de l'étage supérieur, dans une scène où les tensions sont graduellement augmentées, elle est soudainement attaquée par une vague d'oiseaux. Ils sont principalement des mouettes, comme celle qui l'avait blessée dans le bateau. Ce coup avait été un choc momentané pour nous préparer à la séquence plus longue qui se déroule maintenant. Comme dans Sabotage la scène du grenier dure plusieurs minutes. Elle se compose d'un grand nombre de gros plans discontinus organisés afin de simuler la continuité. Par son pouvoir sensoriel et la force rythmique, elle marque un climax émotionnel dans le récit filmique.

Il y eu des analystes qui ont «détesté» la manière dont Hitchcock utilisait la caméra, pour conclure que «ses artiffices restent des artiffices, peu importe s'ils sont merveilleusement conçus et exécutés ${ }^{19}$ et que la pureté artistique demandée par Hitchcock est intrinsèquement déshumanisante. Hitchcock a essayé de défendre ses films disant qu'ils améliorent la vie sociale et affective des spectateurs sans tomber dans le sensationnalisme excessif. Ces films répondent à un besoin profond et omniprésent que de nombreuses personnes ressentent - le besoin d'être effrayées. Il a comparé ses films aux manèges d'une montagne russe, pour lesquels les gens paient de l'argent afin qu'ils puissent être épouventés dans des conditions où il y a peu ou pas de danger. ${ }^{20}$ Hitchcock défend cette approche en plusieurs endroits, mais plus explicitement dans un essai intitulé Le Plaisir de la peur, où il affirme que, bien que peu d'entre nous cherchent la peur «dans le sens réel ou persona», des millions le font indirectement dans l'ambiance obscure d'un théâtre ou du cinéma: Ils s'identifient à des personnages fictifs qui vivent la peur, et ainsi les spectateurs vivent, eux-mêmes, les mêmes sensations de la crainte (le pouls accéléré, l'alternance de sec à humide des palmes, etc), mais sans risquer leur vie. ${ }^{21}$

Metz semble justifier la conception hitchcockienne quand il parle «des pulsions scopique et invocante» qui mobilisent la vue et l'ouïe, des sens qui sont plus marqués du côté de l'imaginaire que le toucher, le goût ou l'odorant. Ces pulsions, comme les autres pulsions sexuelles, peuvent se satisfaire en-dehors de leur

\footnotetext{
${ }^{19}$ François Truffaut, op. cit., p. 25.

${ }^{20}$ Sidney Gottlieb, cité par Singer, op. cit., p. 25.

${ }^{21}$ Sidney Gottlieb op. cit., p. 186.
} 


\section{"Mircea cel Batran" Naval Academy Scientific Bulletin, Volume XIX - 2016 - Issue 1 \\ Published by "Mircea cel Batran" Naval Academy Press, Constanta, Romania // The journal is indexed in: PROQUEST / DOAJ / DRJI / JOURNAL INDEX / I2OR / SCIENCE LIBRARY INDEX / Google Scholar / Crossref / \\ Academic Keys / ROAD Open Access / OAJI / Academic Resources / Scientific Indexing Services / SCIPIO}

objet par la sublimation et sans mettre le spectateur en danger immédiat. ${ }^{22}$ Pour mieux expliquer la notion de jouissance de la peur qui sous-tend l'esthétique hitchcockienne et apparemment l'objectif moral dans l'art cinématographique, Hitchcock met l'accent sur le besoin humain d'expérimenter de telles réactions dans une situation qui n'implique pas vraiment des souffrances. Mais on pourrait se demander: «De la souffrance et des dommages causés à qui?» Sans compter les méchants qui finissent mal dans ses films, les héros d'Hitchcock ou les personnages sympathiques avec qui nous nous identifions souffrent souvent beaucoup. Comme les protagonistes de la tragédie grecque, ils subissent de la détresse réelle et nuisible. Si le public achète des billets, il le fait dans l'espoir de jouir de la vue des événements effrayants affichés là sur l'écran.

En ce qui concerne la nature de la réaction, qu'Hitchcock était si habile à obtenir avec ses films, on doit noter qu'assister à un film, même un «thriller», n'est pas le même qu'être confronté à des dangers véritables dans le monde réel. L'idée centrale quant aux activités comme marcher sur une corde raide, la course automobile, l'alpinisme ou la chasse au gros gibier - pour mentionner les autres exemples d'Hitchcock - est le fait que, ces activités provoquent de la peur en elles-mêmes. Ils cultivent des sentiments de crainte qui peuvent être surmontés, et même appréciés, bien que les conditions dans lesquelles ils se passent sont vraiment dangereuses. Cependant, quand on regarde un film, nous entrons dans un autre genre de circonstance. II n'y a rien de fondamentalement effrayant ou immédiatement dangereux. Nous savons que nous pouvons sortir de la salle à n'importe quel instant si on le désire. Nous savons aussi que les personnages ne le peuvent pas quoiqu'ils nous ressemblent beaucoup. Dans le cadre de leur monde imaginaire, ils sont obligés de faire face à une réalité sombre, de laquelle ils seront ou pas en mesure de s'échapper. Même lorsque les spectateurs se sentent excités par les événements qui rendent le film passionnant, les deux types d'excitation ne sont pas les mêmes.

Tout au long de la représentation, le spectateur est là pour passer un bon moment, alors que les personnages, dans leur propre réalité, pourraient passer par l'enfer. Pour cette raison, la jouissance de la peur éprouvée par le spectateur à laquelle Hitchcock se réfère est une jouissance de la peur de quelqu'un d'autre, des personnages fictifs qui représentent des gens qui semble pourtant être bien réels. Les spectateurs peuvent se sentir

\footnotetext{
${ }^{22}$ Christian Metz, op. cit., p. 17.
}

menacés par les dangers courrus par les personnages si fortement qu'ils expériencent une peur comparable à celle de l'intérieur du film. II s'agit ici d'une illusion. Christian Metz préfère le terme impression à celui d'illusion, car, dit-il, il n'y a de vraie illusion que dans le rêve. Le cinéma tend à l'illusion de réalité, notamment dans certaines fictions, mais il reste à la limite. ${ }^{23}$

Il faut noter ici que l'intention d'Hitchcock de susciter et d'orienter les sentiments de son public sont des réminiscences de la conception aristotelicienne de la catharsis. Douchet remarque qu'il existe une fin morale du suspense hitchcockien, qui consiste à nous «dépiauter d'une enveloppe trompeuse et traîtresse. Lorsqu'enfin elle est complètement détachée de nous, nous pouvons la regarder lucidement et nous constatons à quel point notre vision courante truque, modifie et détourne, pour son unique satisfation le monde naturel; à quel point, donc, elle engage notre responsabilité.» ${ }^{24}$ Même si Hitchcock s'est défendu contre ceux qui lui ont prêté des intentions morales profondes, il est en effet indéniable que parmi les cinéastes qui ont abordé le film noir, il est l'un des seuls qui ait élaboré «tout un univers moral. La forme ici n'enjolive pas le contenu; elle le crée. Tout Hitchcock tient en cette fromule» ${ }^{25}$

Dans sa Poétique, Aristote designe la terreur et la pitié comme la réaction appropriée du public à la tragédie - pour laquelle nous pouvons substituer le drame hitchcockienne. Le sort d'Fipdipe, dit Aristote, évoque de la terreur en nous, parce que nous craignons que des souffrances similaire puissent nous atteindre. ${ }^{26}$ Dans ses références au suspense resultant de notre identification avec un personnage qui court des dangers imminents, Hitchcock aurait pu dire la même chose. Ils se seraient aussi mis d'accord en ce qui concerne les sentiments de la pitié (ou de la compassion). Les drames d'Hitchcock sont tellement tendus, pleins d'actions qui suscitent des réactions, de telle manière que les sentiments de la pitié et de la compassion ne peut y avoir qu'un rôle essentiel. En plus, Hitchcock est en mesure de déployer ces sentiments comme les moteurs qui propulsent notre implication affective dansl'intrigue.

Quand Cary Grant et Eva Marie Saint dans Nord par Nord-Ouest ont appris la vérité l'un sur l'autre, mais doivent être séparés de sorte qu'elle puisse accomplir sa mission en tant qu'agent secret, nous sympatisons avec eux, mais seulement

\footnotetext{
${ }^{23}$ Christian Metz, op. cit., p. 133.

24 Jean Douchet, op. cit., p. 92-93.

${ }^{25}$ Claude Chabrol, p. 154.

${ }^{26}$ Aristote, op. cit., ch. XIII.
} 


\begin{abstract}
"Mircea cel Batran" Naval Academy Scientific Bulletin, Volume XIX - 2016 - Issue 1
Published by "Mircea cel Batran" Naval Academy Press, Constanta, Romania /I The journal is indexed in: PROQUEST / DOAJ / DRJI / JOURNAL INDEX / I2OR / SCIENCE LIBRARY INDEX / Google Scholar / Crossref /

Academic Keys / ROAD Open Access / OAJI / Academic Resources / Scientific Indexing Services / SCIPIO
\end{abstract}

brièvement. Grant doit être frappé de façon à ce qu'elle puisse aller accomplir sa mission; quant à lui, il $\mathrm{a}$, dans son destin héroïque, la mission de parvenir à la maison sur le mont Rushmore par lui-même

afin de la sauver, et ainsi de suite, selon que le cours du suspense incessant le demande.

Dans d'autres films, il ya parfois plusieurs possibilités de pitié et de compassion, de sympathie ou d'autres sentiments, comme dans Les enchaînés (Notorious), lorsque'on regarde le déclin de la santé et de la beauté d'Ingrid Bergman, après qu'elle a été empoisonnée, ou dans L'homme qui en savait trop lorsque la mère du garçon est presque folle parce que son fils a été enlevé. Toutefois, ce qui prédomine est généralement la nécessité de passer d'un développement passionnant à l'autre dans leur progression sérielle, mais aussi nterdépendante.

En parlant de ses films dans ses interviews, Hitchcock mentionne les différences entre un film de suspense, un thriller, un mystère, et un whodunit, et dans l'un de ses articles, il affirme que ses films ne sont pas des films d'horreur. II est important de voir comment ces structures filmiques fonctionnent pour mieux comprendre quelques notions clés de la conception hitchcockienne du plaisir dramatique.

Décrivant le premier de ces cinq genres, il parle de la différence entre le suspense et la surprise. II nous demande d'imaginer deux hommes qui sont assis à une table: «Nous sommes en train de parler, il y a peut-être une bombe sous cette table et notre conversation est très ordinaire, il ne se passe rien de spécial, et tout d'un coup: boum, explosion. Le public est surpris, mais, avant qu'il ne l'ait été, on lui a montré une scène absolument ordinaire, dénouée d'intéret. Maintenant, examinons le suspense. La bombe est sous la table et le public le sait, probablement parce qu'il a vu l'anarchiste la déposer. Le public sait que la bombe explosera à une heure et il sait qu'il est une heure moins le quart - il y a une horloge dans le décor; la même conversation anodine devient tout à coup très intéressante parce que le public participe à la scène. Il a envie de dire aux personnages qui sont sur l'écran: «Vous ne devriez pas raconter des choses si banales, il y a une bombe sous la table, et elle va bientôt exploser. » Dans le premier cas, on a offert au public quinze secondes de surprise au moment de l'explosion. Dans le deuxième cas, nous lui offrons quinze minutes de suspense. La conclusion de cela est qu'il faut informer le public chaque fois qu'on le peut, sauf quand la surprise est un twist, c'est-à-dire lorsque l'inattendu de la conclusion constitue le sel de l'anecdote. ${ }^{27}$

Ceci est paradigmatique du suspense hitchcockien. Il exige de nous, les spectateurs et de notre imagination de connaître à l'avance le danger de la situation. Nous éprouvons un sentiment d'anxiété pour les personnages, dit Hitchcock, même si les personnages ne réalisent pas le risque qu'ils courrent. Selon Hitchcock, pour qu'il y ait du suspense, il est crucial que la bombe n'explose pas. Si cela se produisait, on aurait rien à attendre avec appréhension. Le public peut subir le choc, mais le suspense en tant qu'effet cinématographique n'existerait plus. Ce point est important pour l'auto-critique qu'Hitchcock se fait pour avoir laissé le garçon dans Sabotage mourir quand la bombe explose. II sent rétrospectivement qu'il a violé le principe du suspense, et il remarque également que la structure entière du suspense a été affectée par cette décision.

À l'opposé du film de suspense, Hitchcock met le whodunit. Dans ce genre, le public n'a pas de connaissance préalable des faits de l'intrigue. Les détails pertinents sont présentés à la fin, non pas au début. Les indices sont là dès le début, ils intriguent le spectateur mais ne créent pas de suspense. Bien qu'Hitchcock ne dise pas grand chose sur son propre emploi des effets du whodunit, elles servent souvent comme des éléments principaux dans ses films. Ils se produisent dans la recherche de l'homme coupable de Jeune et innocent qui clignote ses yeux mais est par ailleurs inconnu, dans Spellbound ou l'assassin apparaît dans le rêve troublé de Gregory Peck, en restant quand même toujours méconnaissable, dans Mais qui a tué Harry pour la personne qui a occasionné la mort, dans Fenêtre sur cour pour la cause de la disparition suspecte de la femme malade, etc. Les morceaux de données sur des homicides, comme c'est généralement le cas, sont fondamentaux pour l'histoire, mais c'est seulement à la fin que nous apprenons ce qu'ils signifient, et qui avait commis le crime.

En extrapolant au-delà de quelques remarques faites par Hitchcock, on peut envisager le mystère comme le récit de certains accidents inconnus ou on peut avoir de la criminalité ou pas. Dans Spellbound nous sommes intrigués par l'étrange comportement illégal de Gregory Peck et de son agonie cachée, dont Ingrid Bergman doit essayer de découvrir la source. Elle est une psychiatre, mais son expertise se révèle comme une forme de travail de détective. James Stewart dans Vertigo, qui est un détective ayant besoin d'aide

\footnotetext{
${ }^{27}$ François Truffaut, op. cit., pp. 58-59.
} 


\begin{abstract}
"Mircea cel Batran" Naval Academy Scientific Bulletin, Volume XIX - 2016 - Issue 1
Published by "Mircea cel Batran" Naval Academy Press, Constanta, Romania /I The journal is indexed in: PROQUEST / DOAJ / DRJI / JOURNAL INDEX / I2OR / SCIENCE LIBRARY INDEX / Google Scholar / Crossref /

Academic Keys / ROAD Open Access / OAJI / Academic Resources / Scientific Indexing Services / SCIPIO
\end{abstract}

psychiatrique lui-même, vise à clarifier les activités de la bizarre Kim Novak. Les scènes qui constituent le film sont comme «les pièces d'un puzzle: chacune constitue un morceaux de savoir, mais reste insignifiante tant que la nature du tout dont ils font partie ne sera pas clairement établie.» ${ }^{28}$ Des éléments mystérieux, comme l'expression «39 marches» ou le sens caché de pi dans Torn Curtain, envahissent souvent les films de mystère d'Hitchcock et s'entrecroisent facilement avec le suspense qui résulte lorsqu'on essaie de découvrir leurs sens.

Le thriller utilise le suspense mais il le fait pour une fin qui va au-delà de celui-ci. Le suspense est un état affectif qui passe de la terreur au soulagement. Le suspense suscite la curiosité pour un problème qui a été posé à propos d'un événement étrange et de ses conséquences telles qu'elles se déroulent dans l'intrigue, il attire aussi le souci pour le bien-être des personnages de la fiction que nous trouvons intéressants pour quelque raison que ce soit, ou tout simplement il marque l'implication esthétique dans une histoire fascinante. Pour qu'un film soit un thriller, toutefois, ou pour qu'il ait les ingrédients distinctifs d'un, il doit vivement reveiller notre expérience sensorielle par l'excitation soigneusement conçue qui se produit lors des moments cruciaux dans le récit. Compte tenu de la nature du film, il s'agit des composants visuels, auditifs, et parfois kinesthésiques de notre conscience. Les images atteignent nos sens et activent nos organes d'une manière qui peut être désagréable pour le moment mais tout à fait acceptable et même bienvenue grace à l'artifice ingénieux qui nous permet de jouir du film. Le suspense joue sur le fait que, dans un contexte adéquat, tout le monde aime tréssaillir. Le meilleur exemple de ce genre est Psycho.

Les films d'horreur sont tout à fait différents des thrillers. En 1936 dans un essai intitulé Why Thrillers Thrive, Hitchcock contraste les deux. II associe aux thrillers des motifs moraux ainsi qu'esthétiques. II dit que notre état naturel est tel que nous avons besoin d'une secousse si non, nous devenons mous et gélifiés. Comme l'existence civilisée ne donne pas suffisamment de sensations fortes, nous devons les expérimenter artificiellement, et l'écran est le meilleur moyen pour cela. Surtout s'il s'agit de ce que Hitchcock appelle un film bien fait, un thriller peut déterminer les spectateurs de se sentir comme s'ils participaient aux événements fascinantes, tout en sachant qu'ils ne sont pas en danger. Mais le film d'horreur met trop d'effort en ce sens et devient donc répulsif, insiste Hitchcock.

${ }^{28}$ Jean Pierre Esquenazi, 2001, p129.

DOI: 10.21279/1454-864X-16-I1-084
Hitchcock trouve artificielle l'émotion crée par ce type de film: «Le terme [horreur] qui veut dire extrême aversion», a été en gros appliqué aux films qui offrent une secousse émotionnelle, qui exploitent le sadisme, la perversion, la bestialité, et la déformation. [. . . ] C'est tout à fait érroné! Etant vicieux et dangereux, il est admissible pour un film d'être horrifique, mais pas horrible; entre les deux il y a une ligne de séparation évidente.» ${ }^{29}$

Par cette distinction, Hitchcock semble invoquer les critères du bon goût ou de ce qui est moralement admissible. Ce serait interessant d'observer ici les échos de la théorie aristotellicienne du plaisir convenable. Tout au long de son travail, Hitchcock invoque souvent un sentiment d'horreur, sans lui permettre de transformer ses thrillers en films d'horreur. Nous sommes soumis à un sentiment d'effroi quand Cary Grant est mitraillé dans un champ de maïs dans North by Northwest, quand il conduit ivre au bord d'une falaise, vers le début de ce film et, plus tard, quand lui et Eva Marie Saint pendent d'une autre falaise. Nous éprouvons des sentiments semblables quand des aggressions à coup de couteau, soudains et mortels se produisent dans les films hitchcockiens. Dans le même temps, il est également vrai qu'Hitchcock emploie l'élément d'horreur seulement dans des paramètres spéciaux et limités ou il peut avoir son plus grand effet. Dans le film d'horreur archétypal dont Hitchcock parle, l'impact émotionnel est souvent altéré, et d'habitude lui manque la qualité esthétique, précisément parce qu'il est exagéré.

A côté de la théorisation de ces cinq genres, il existe, dans la méthodologie d'Hitchcock la survenance d'une tentative d'intégrer et d'harmoniser les valeurs de tous. II insiste sur le fait que le réalisateur doit toujours choisir entre le suspense et le choquant, voir la surprise. Les deux variantes suivent des voies différentes et sont incompatibles à tout moment dans le film. Étant fondé sur une connaissance préalable du public, le suspense est incapable de créer la secousse qui vient de l'inattendu de la surprise ou du choc. Résultant d'une poussée soudaine sur nos facultés sensorielles, le choc, élimine toute possibilité de suspense simultané. En combinant les deux dans l'ensemble du film, Hitchcock remarque qu'il consacre les deux tiers du film au suspense et un tiers seulement aux événements choquants.

Dans deux de ses essais, Hitchcock invoque le concepte de Dieu comme un moyen de présenter des idées, non pas de l'origine ou de la

\footnotetext{
${ }^{29}$ Sidney Gottlieb op. cit., p. 288 (la traduction de l'auteur).
}

(c) 2015. This work is licensed under the Creative Commons Attribution-Noncommercial-Share Alike 4.0 License. 


\section{"Mircea cel Batran" Naval Academy Scientific Bulletin, Volume XIX - 2016 - Issue 1 \\ Published by "Mircea cel Batran" Naval Academy Press, Constanta, Romania /I The journal is indexed in: PROQUEST / DOAJ / DRJI / JOURNAL INDEX / I2OR / SCIENCE LIBRARY INDEX / Google Scholar / Crossref / \\ Academic Keys / ROAD Open Access / OAJI / Academic Resources / Scientific Indexing Services / SCIPIO}

signification de l'univers, mais plutôt sur la nature du suspense comme il existe dans ses films. Dans Let 'Em play God, il renforce son point de vue sur la précognition du public, élément indispensable pour qu'il y ait du suspense. Les spectateurs jouent Dieu dans la mesure où ils savent à l'avance quels sont les dangers. ${ }^{30}$ Mais, dans Would you like to know your future? Hitchcock donne à penser que si nous avions connaissance de notre l'avenir, «plus de joie sortirait de la vie. [car] l'inconnu est attirant précisément parce qu'il est mystérieux.» ${ }^{31}$

Le premier essai est d'intérêt parce que quelqu'un qui fait des films comme Hitchcock joue également Dieu. En formulant son argument, il se réfère à la structure de Corde. Dans ce film, qui traite de la mentalité de deux jeunes hommes qui jouent Dieu, en décidant de mettre fin à la vie de leur ami. Les spectateurs savent dès le début qui a commis le meurtre. Néanmoins, nous ne savons pas comment et si les auteurs seront arrêtés. Notre sentiment de suspense résulte de là plutôt que de la precognition. Le deuxième essai, écrit onze ans plus tard, est encore plus problématique. Ici Hitchcock dit que Dieu est miséricordieux et nous épargne de l'ennui et de la futilité d'une vie sans suspense. II semble interpréter la motivation divine comme un modèle de ce que les cinéastes comme lui-même ont essayé d'effectuer. Sa déclaration peut être vue comme une autre justification de sa mission comme artiste.

Aussi importante que le suspense, sinon plus importante, est la libération du suspense, synonyme de la catharsis aristotelicienne. En représentant le développement et la libération de la tension comme la base du suspense, Hitchcock note également l'avantage du contrepoint pour alléger la lourdeur des événements mélodramatiques avec des variations dans l'atmosphère générale. Deux éléments contribuent aux effets contrastés de ce genre: le portrait réaliste de la vie et l'inclusion de l'humour dans le mélodrame. Parfois le contenu du film est sérieux et le regard en est humoristique. D'ailleurs, Hitchcock avoue qu'au moment où lui et son équipe écrivent un scénario, la phrase qui revient le plus souvent est : «Est-ce que ce serait amusant de le faire assassiner de cette façon ? » $^{32}$ On trouve cet aspect dans Psycho et les Oiseaux, qui ont des intrigues sérieuses racontées avec ironie.

Hitchcock offre, lui-même, une interprétation plausible de sa technique quand il parle de

\footnotetext{
${ }^{30}$ Sidney Gottlieb op. cit., p. 118

${ }^{31}$ Ibidem.

${ }^{32}$ François Truffaut, op.cit., p. 168.
}

l'orchestration cinématographique, du contrepoint, voire analogue à ce qui se passe dans la création et l'exécution de la musique. L'analogie d'Hitchcock semble juste. La grande vertu des approches formalistes du film réside dans la compréhension de la manière dont les innovations techniques contribuent à une dynamique interactive au sein de chaque oeuvre. L'interaction se produit dans une réceptivité continue parmi les composants cinématographiques qui est en effet similaire aux modèles harmoniques qui constituent l'art musicale. La mise ensemble de «petits morceaux de montage est une partie de la panoplie compositionnelle du film.» ${ }^{33}$ Et au-delà, l'intrigue elle-même est normalement une représentation narrative des différentes possibilités que la vie donne, des intérêts et des expériences variés que les êtres humains peuvent rencontrer, chacun d'entre eux générant une variété de problèmes qui doivent être résolus.

Au fur et à mesure que les solutions possibles se développent dans l'intrigue, les personnages, qui sont à part dans la poursuite, réagissent systématiquement les uns aux autres. C'est la que se trouve la source de la progression dramatique, qui est capable de susciter des sentiments et de créer le suspense dans les téléspectateurs qui regardent, mais qui ne peuvent pas participer. Le ricochet soutient notre attention d'un moment à l'autre de l'histoire, et cela nous permet de nous identifier avec les personnages qui vivent dans leur monde fictive.

Dans les mains d'un maître comme Hitchcock, l'inventivité technique ressemble la créativité de la composition musicale. Hitchcock peut orchestrer ces effets, le plus inventivement possible par l'intelligence des mouvements de sa caméra qui se déplace d'une perspective à l'autre. Dans tous ces films, le magnifique sens du rythme d'Hitchcock est la clé de son succès. Le rythme est assez lent pour susciter en nous un sentiment de ce qu'est la vraie vie et assez rapide, pour nous préparer pour l'accélération qui entraîne bientôtaususpense

et parfois à la terreur. Cette acuité de ce qui est esthétiquement disponible à travers le temps luimême, par le biais de la simple durée de l'existence banale, devient une technique qu'Hitchcock utilise beaucoup dans ses films. Elle est, remarque Singer, parallèle à celle utilisée par Mozart au début de ses concertos pour le piano, où seulement de la musique discrète se fait entendre jusqu'à ce que le solo de l'instrument entre, à quel point celui-ci et la accompagnement orchestral commencent à moduler largement. ${ }^{34}$

\footnotetext{
${ }^{33}$ Irving Singer, op. cit., p. 39.

${ }^{34}$ Irving Singer, op. cit., p. 52.
} 
"Mircea cel Batran" Naval Academy Scientific Bulletin, Volume XIX - 2016 - Issue 1

Published by "Mircea cel Batran" Naval Academy Press, Constanta, Romania /I The journal is indexed in:

PROQUEST / DOAJ / DRJI / JOURNAL INDEX / I2OR / SCIENCE LIBRARY INDEX / Google Scholar / Crossref /

Academic Keys / ROAD Open Access / OAJI / Academic Resources / Scientific Indexing Services / SCIPIO

Hitchcock affiche une conscience rythmique comparable à celle que tous les musiciens ont accompli. Il est le maître dans le domaine de la représentation visuelle grace au sens du rythme car chez lui « les séquences d'un film ne doivent jamais piétiner, mais toujours aller de l'avant, exactement comme un train avance roue derrière roue, ou, plus exactement encore, comme un train « à crémaillère gravit le chemin de fer de montagne cran par cran. » ${ }^{35}$

Hitchcock décrit aussi son intérêt pour le matériel, en mettant l'accent sur les objets afin d'accroître l'effet de réalité, il affirme que ceux-ci l'aident à surmonter l'inexpressivité du visage humain. Cette approche aux choses, aux objets matériaux comme la base de la construction de la scène, est entièrement cohérente avec l'intérêt d'Hitchcock pour la construction d'un film par le biais du montage. II sous-tend également sa conception de la façon dont un réalisateur devrait traiter les acteurs. Ils sont les convoyeurs de la signification psychologique sans laquelle il ne peut y avoir de tension dramatique ou de participation émotionnelle de la part du public. En tant que personnages, les acteurs nous représentent. C'est pourquoi nous vivons indirectement l'expérience qu'ils vivent dans le récit et pourquoi nous sommes capables de nous inquiéter pour eux et même de nous identifier avec eux. Et pourtant, «le montage dans le cinéma comme le cubisme en peinture - brise l'image des personnalités variables des personnages et impose des perspectives visuelles qui contreviennent du point de vue formel à ce que nous considérons être leur réalité. ${ }^{36}$ Loin de penser qu'il s'agit là d'une lacune inhérente à la technique cinénematographique, Hitchcock réjouit de la liberté de l'imagination et de la créativité qu'elle permet au cinéaste. Elle montre l'inventivité du « cinéma pur» et incarne la différence entre l'écran et la scène théâtrale.

Les pièces de théatre doivent également subordonner la personnalité de l'acteur aux exigences de la fiction. Mais quel que soit le sens de la pièce, elle est toujours transmise par une personne qui est là vive et mobile en face de nous. Au cinéma cette personne a été transformée en un objet matériel, une concoction d'images produites par une machine ou plusieurs, et projetée sur une surface bidimensionnelle. On pourrait accepter cet état de choses comme une constante essentielle dans cette forme d'art et d'essayer de traiter avec lui du mieux que l'on

\footnotetext{
${ }^{35}$ François Truffaut, op.cit., p. 57.

${ }^{36}$ Irving Singer, op. cit., p. 59(la traduction de l'auteur).
}

peut. Mais ayant été le suprême technicien, qu'il a été, Hitchcock a vu la situation un peu différemment. Cette situation lui a offert la possibilité de faire quelque chose qu'aucun acteur ne pourrait représenter ou exprimer par lui-même. C'est pourquoi Hitchcock insiste sur le fait que dans ses films il ne peut y avoir de place pour le «virtuoso acteur», celui qui pense que seuls ses talents d'artiste lui permettent d'atteindre le public. «L'acteur de l'écran doit beaucoup plus plastique», dit Hitchcock, il doit se laisser être utilisé par le réalisateur et la caméra., II est demandé surtout de laisser la caméra ajouter la plupart des accents et d'emphases. ${ }^{37}$

Néanmoins, Hitchcock savait que, avec les stars comme couverture, les réalisateurs et les producteurs pouvaient s'en tirer avec beacoup d'innovations vers lesquelles leur goût artistique les poussait. Les stars ne doivent pas dominer une production, mais Hitchcock souligne leur importance comme des aimants qui gagnent le public qui s'identifie avec eux dans le film. Les stars peuvent servir d'aimants par leur capacité d'incarner l'esprit humain, son visage et sa voix avec lequel tous les spectateurs veulent prendre contact par l'intermède de l'expérience le filmique.

\footnotetext{
${ }^{37}$ François Truffaut, op.cit., p. 27.
} 
"Mircea cel Batran" Naval Academy Scientific Bulletin, Volume XIX - 2016 - Issue 1

Published by "Mircea cel Batran" Naval Academy Press, Constanta, Romania // The journal is indexed in:

PROQUEST / DOAJ / DRJI / JOURNAL INDEX / I2OR / SCIENCE LIBRARY INDEX / Google Scholar / Crossref /

Academic Keys I ROAD Open Access / OAJI / Academic Resources / Scientific Indexing Services / SCIPIO

\section{CONCLUSIONS}

Notre article fait l'analyse de l'œuvre cinématographique d'Hitchcock et de la manière qu'il s'est servi des techniques cinématographiques pour offrir au public le plus grand plaisir et ainsi assurer le succès de ses films. Nous nous sommes appuyé dans notre recherche sur les interviews et les déclarations d'Hitchcock et sur la critique qui lui a été dédiée. Pour Hitchcock l'art cinématographique constitue le véhicule du plaisir resultant de l'identification avec un personnage, de l'émotion, du suspense, et, de manière explicite, de la structure du montage. Hitchcock est en effet un des réalisateurs qui ont suivi les principes du plaisir dramatique d'une manière exceptionnelle afin de créer le suspense, c'est-à-dire susciter des sensations fortes chez le spectateur pour le contraindre à rester calé dans son fauteuil et à se laisser emporter jusqu'à la fin. En d'autres termes, le suspense hitchcockien, comme inspiré de la mécanique de la tragédie grecque, est une combinaison du danger imminent, de la position supérieure du public, de l'identification avec le personnage qui favorise l'expérience des émotions fortes comme la peur, la pitié, l'angoisse etc., tout ça bien consolidé par la structure du récit.

\section{BIBLIOGRAPHIE}

[1] BELLOUR, Raymond. L'analyse du film, Ed. Albatros, Paris, 1979.

[2] CHABROL, Claude; ROHMER, Eric, Hitchcock, Editions Ramsay, 2006.

[3] DOUCHET, Jean, Cahiers du Cinéma, 1999.

[4] EISENSTEIN, Sergei, Articole alese, Ed. Cartea Rusă, Moscova, 1958.

[5] ESQUENAZI, Jean Pierre, Hitchcock et l'aventure de Vertigo. L'invention à Hollywood, CNRS Editions, Paris, 2001.

[6] FABE, Marilyn, Closely Watched Films. An Introduction to the Art of Narrative Film Technique, University of California Press, Los Angeles, 2004.

[7] SINGER, Irving, Three Philosophical Filmmakers Hitchcock, Welles, Renoir, the MIT Press, Cambridge, Massachusetts, 2004.

[8] TRUFFAUT, François, Hitchcock, Editions Ramsay, 1983.

\section{LIENS ÉLECTRONIQUES:}

[1] ARISTOTE, La Poétique. Chapitre XIV, traduction Charles Batteux, Ed. J. Delalain, 1874, Paris (http://agora.qc.ca)

[2] BAZIN, André, Ce este cinematograful ? Traducere din limba franceză şi prefaţă de Ervin Voiculescu (http://d.scribd.com)

[3] DUROZOI, Gérard ; André Roussel, Dictionnaire de philosophie, Éditions Nathan, Paris, 2003 (http://www.cafe-philo.eu)

[4] METZ, Christian, Le signifiant imaginaire - Psychanalyse et cinéma, Ed : Union Générale d'Editions (http://www.idixa.net) 